\section{In response (2)}

We thank Wallis and Guptha for sharing their audit results. Their results show that the majority of their patients were admitted with an infection, which was also stated as the cause of death. Our results revealed that infection was the primary reason for admission in $32 \%$ of the cohort. ${ }^{1}$ Symptoms arising as a result of infection can be similar to those of someone who is dying, and as healthcare professionals working in an environment which focuses on cure, we sometimes treat infections without recognising that the patient is actually dying from their underlying illness. ${ }^{2-4}$

However, we fully agree that the treatment of an infection can be entirely appropriate for patients who may be entering their last days of life - either for symptom control or because the prognosis is uncertain (especially in those with non-malignant disease who have unpredictable disease trajectories). The current version of the Liverpool Care Pathway (LCP) for the dying does not stipulate that all antibiotics should be stopped, but that inappropriate antibiotics should be discontinued. ${ }^{5}$ Thus in those patients where appropriate, it is possible for the patient to be on the LCP while receiving antibiotics. However, as suggested by our audit and the work of others, the 'diagnosis of dying' can be difficult to make and thus the 'right time' to place a patient onto the LCP or other end-of-life care pathway can be challenging. We therefore advocate that until sensitive and specific prognostication tools are available, as stated above, we should 'assist clinicians in identifying those patients who might die during their current hospital admission thereby enabling active treatment where appropriate alongside symptom relief'. ${ }^{1}$ This approach would enable those patients who require appropriate antibiotics to receive them, while allowing 'healthcare professionals to allay their own anxieties in instances of clinical uncertainty?

JANE GIBBINS

Specialist Registrar in Palliative Medicine

RACHEL MCCOUBRIE

Consultant in Palliative Medicine

KAREN FORBES

Macmillan Professorial Teaching Fellow and Consultant in Palliative Medicine

University Hospitals of Bristol NHS Foundation Trust

\section{References}

1 Gibbins J, McCoubrie R, Alexander N, Kinzel C, Forbes K. Diagnosing dying in the acute hospital setting; are we too late? Clin Med 2009;4:116-9.

2 SUPPORT Principle Investigators. A controlled trial to improve care for seriously ill hospitalised patients: the study to understand prognoses and preferences for outcomes and risks of treatment. JAMA 1995;274:1591-8.

3 Ellershaw J, Ward C. Care of the dying patient: the last hours or days of life. BMJ 2003;326:30-4.

4 Miller F, Fins J. A proposal to restructure hospital care for dying patients. $\mathrm{N} \mathrm{Engl}$ Med 1996;334:1740-2.

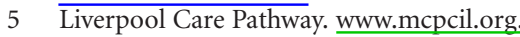
uk/liverpool_care_pathway

\section{Investigation of the patient with pleural effusion (1)}

Editor - Rahman and Munavvar's paper on investigating the patient with pleural effusion (Clin Med April 2009 pp 174-8) made no mention of the utility of quantifying pleural fluid adenosine deaminase (ADA) as a diagnostic parameter. Assay of ADA is simple and inexpensive, coupled with a relatively high sensitivity and specificity in identifying tuberculous pleuritis, enhanced further when the effusion in question is richly lymphocytic. ${ }^{1,2}$ While the positive predictive value of ADA may be lowered by its use in areas of low tuberculosis (TB) prevalence, its negative predictive value should remain unaffected, retaining its overall value as a subsidiary 'rule out' test for suspected TB pleural disease. ${ }^{3}$ Furthermore, ADA levels falling below the diagnostic cut-off threshold (around $40 \mathrm{U} / \mathrm{l}$ ) have been shown to virtually exclude TB as a cause of pleural effusion even when the collection is lymphocytic. ${ }^{3,4}$ We suspect that many physicians continue to find the analysis of ADA useful in the setting of suspected TB pleural sepsis while eagerly awaiting the emergence of newer immune-based tests of pleural fluid.

FELIX CHUA

Consultant Thoracic Physician

JANICE GROTH

Senior TB Specialist Nurse,

St George's Healthcare NHS Trust, London

\section{References}

1 Valdes L, Alvarez D, San Jose E et al. Value of adenosine deaminase in the diagnosis of tuberculous pleural effusion in young patients in a region of high prevalence of tuberculosis. Thorax 1995;50:600-3.

2 Burgess LJ, Maritz FJ, Le Roux I et al. Combined use of adenosine deaminase with lymphocyte/neutrophil ratio. Chest 1996;109:414-9.

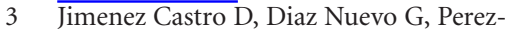
Rodriguez E, Light RW. Diagnostic of adenosine deaminase in nontuberculous lymphocytic pleural effusions. Eur Respir J 2003;21:220-4.

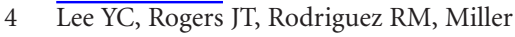
KD, Light RW. Adenosine deaminase levels in nontuberculous lymphocytic pleural effusions. Chest 2001;120:356-61.

\section{Investigation of the patient with pleural effusion (2)}

Given the fact that culture of pleural fluid (using Lowenstein medium) has only 36.6\% sensitivity for diagnosis of tuberculous pleural effusion versus $79.8 \%$ sensitivity obtained with the more invasive modality of histological identification of caseating granuloma, ${ }^{1}$ among the non-invasive 'special tests' for evaluation of pleural effusion, ${ }^{2}$ mention should also have been made of adenosine deaminase (ADA) ${ }^{3}$ and interferon-gamma (IFN-gamma), ${ }^{4}$ so as to complement strategies such as staining and culture for acid-fast bacilli. ${ }^{2}$ In a systematic review of 63 studies ADA was characterised by positive likelihood ratio 9.03 (95\% confidence interval (CI) 7.19 to 11.35 ), negative likelihood ration 0.10 (95\% CI 0.07 to 0.14 ) for diagnosis of tuberculous pleural effusion. ${ }^{3}$ Correspondingly, in a systematic review of 22 studies, diagnostic accuracy of IFN-gamma was characterised by positive likelihood ration amounting to 23.45 (95\% CI 17.31 to 31.78 ), and negative likelihood ratio 0.11 (95\% CI 0.07 to 0.16$).{ }^{4}$ In the setting of tuberculous effusion prevalence of $5 \%$, post-test probability of a negative ADA test has been estimate to be $0.4 \%$, increasing to $2.4 \%$ where tuberculous pleural effusion has a $25 \%$ prevalence. For INF-gamma, corresponding post-test probabilities are $0.22 \%$ and $1.2 \%$ respectively.

OSCAR MP JOLOBE

Manchester Medical Society,

Manchester 


\section{References}

1 Valdes L, Alvarez D, Jose ES et al. Tuberculous pleurisy: a study of 254 patients. Arch Intern Med 1998;158:2017-21.

2 Rahman NM, Munavvar M. Investigation of the patient with pleural effusion. Clin Med 2009;9:174-8.

3 Liang QL, Shi HZ, Wang K, Qin SM, Qin XJ. Diagnostic accuracy of adenosine deaminase in tuberculous pleurisy: A meta analysis. Respir Med 2008;102:744-54

4 Jiang J, Shi H-Z, Liang Q-L, Qin S-M, Qin X-J. Diagnostic value of interferon-gamma in tuberculous pleurisy. A meta analysis. Chest 2007;131:1133-41.

5 Greco S, Girardi E, Masciangelo R, Capoccetta GB, Saltini C. Adenosisne deaminase and interferon gamma measurements for the diagnosis of tuberculous pleurisy; a meta analysis. Int J Tuberc Lung Dis 2003;7:777-86.

\section{In response to both}

We would like to thank Drs Chua, Groth and Jolobe for their letters concerning our article on respiratory investigations in pleural effusion, highlighting the use of pleural fluid adenosine deaminase (ADA) as a potential diagnostic test for suspected TB pleuritis. We agree that pleural fluid ADA is an important, useful and inexpensive diagnostic test for the investigation of patients with a moderate to high probability of TB pleuritis, on the basis of the evidence quoted. Pleural fluid ADA was not included in our article due to varying availability in the UK of this test, and constraints on the article length. The majority of chest physicians practise in low prevalence areas in the UK, although there are some areas where this is not the case. Pleural fluid ADA is associated with false positive results (eg pleural infection, rheumatoid pleuritis and malignancy) and in areas of low prevalence such as much of the UK, this limits ADA to being a 'rule out' test. We would suggest ADA is restricted to use in lymphocytic effusions in which there is at least a reasonable pretest probability of TB pleuritis. In addition, the diagnosis of TB pleuritis using markers of immune cell activation (ie ADA or interferon-gamma releasing assays) do not achieve specimens on which microbiological sensitivity may be tested. Although data from the USA sug- gest that the pattern of resistance in $\mathrm{TB}$ pleuritis reflects that seen in local pulmonary $\mathrm{TB}$, this may not be helpful in the assessment of resistance in immigrants or recently returned travellers. ${ }^{1}$ In these circumstances, we would suggest pleural biopsy for histology and culture remains the gold standard diagnostic test, conducted either using Abram's needles or under image/thoracoscopic guidance. We would like to thank the authors for their interest in our article.

NM RAHMAN

MRC Training Fellow and Specialist Registrar, Oxford University

M MUNAVVAR

Consultant Chest Physician, Lancashire Teaching Hospital NHS

Foundation Trust

\section{Reference}

1 Baumann MH, Nolan R, Petrini M et al. Pleural tuberculosis in the United States: incidence and drug resistance. Chest 2007;131:1125-32.

\section{Non-cystic fibrosis bronchiectasis}

Editor - In their excellent review of noncystic fibrosis bronchiectasis Murray and Hill (Clin Med April 2009 pp 164-9) failed to mention an important and increasingly prevalent variety of bronchiectasis in older adults. Bronchiectasis associated with nontuberculous mycobacterial (NTM) infection, notably Mycobacterium avium complex (MAC) and Mycobacterium abscessus, is now frequently seen in older patients, notably women who give no history of respiratory disease earlier in life and are often non-smokers. The disease is characterised by bronchiectasis which most often involves the right middle lobe and the lingular segment of the left upper lobe in association with nodules - small nodules in the 'tree-in-bud' configuration and larger nodules which are often peripheral in their distribution and may occasionally cavitate although cavitation is not a characteristic feature of this disorder. This disorder which has been labelled nodular bronchiectasis may be detected when investigating an older patient with a history of cough and sputum production associated with recurrent infectious exacerbations and, later in the illness, with progressive weight loss. The chest radiograph is usually characterised by large lungs which may be surprisingly unremarkable although in the later stages peripheral nodules and features of bronchiectasis may become apparent. In the earlier stages of the disease a computed tomography chest X-ray is usually striking for its abnormalities, an example of which is included (Fig 1).

Although the study by Jenkins et al suggested poor results when treating NTM lung disease, the majority of their patients with MAC disease had cavitary disease which is recognised to be poorly responsive to treatment. ${ }^{1}$ Their study may thus not be generalisable to patients with nodular bronchiectasis who do seem in case series to respond to treatment. ${ }^{2}$ In our clinic, treatment regimens with azithromycin, ethambutol and clofazimine have been well tolerated and often result in sputum culture conversion and improvement of symptoms. Treatment is also given in the belief that it will arrest the progression of the disease. Azithromycin and clarithromycin are thought to be the only medications which are effective against MAC and it would thus be important not to give one of these antibiotics, as suggested by Murray and Hill, without concurrent medication to patients who might have MAC nodular bronchiectasis for fear of

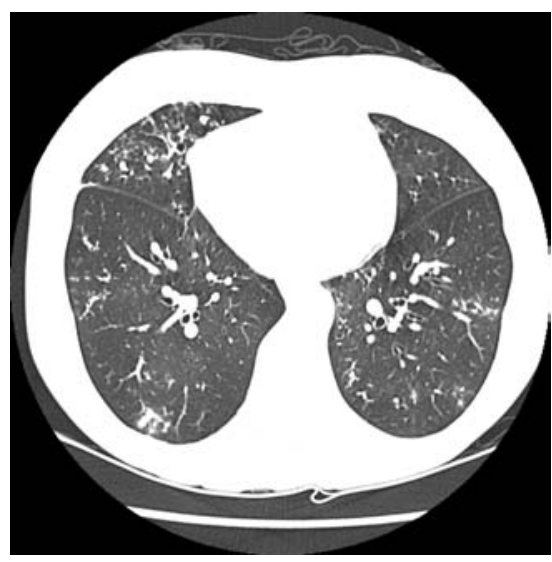

Fig 1. Example of nodular bronchiectasis in a patient with repeatedly positive sputum smear and culture for Mycobacterium avium complex. 